\title{
Light Quark Masses with $N_{f}=2$ Wilson Fermions*
}

\author{
N. Eicker ${ }^{\mathrm{a}}$, Th. Lippert ${ }^{\mathrm{a}}$, B. Orth ${ }^{\mathrm{a}}$ and K. Schilling ${ }^{\mathrm{a}}$

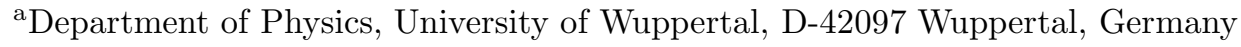

\begin{abstract}
We present new data on the mass of the light and strange quarks from SESAM $/ \mathrm{T} \chi \mathrm{L}$. The results were obtained on lattice-volumes of $16^{3} \times 32$ and $24^{3} \times 40$ points, with the possibility to investigate finite-size effects. Since the SESAM $/ \mathrm{T} \chi \mathrm{L}$ ensembles at $\beta=5.6$ have been complemented by configurations with $\beta=5.5$, moreover, we are now able to attempt the continuum extrapolation $(\mathrm{CE})$ of the quark masses with standard Wilson fermions.
\end{abstract}

\section{Introduction}

The precise determination of light quark masses, $\bar{m}$, from full QCD simulations is of considerable interest since the lattice provides the only known access to their absolute scale.

We have pointed out some time ago [1] that vacuum polarization effects have sizeable impact on extracting $\bar{m}$ from the empirical $m_{\pi} / m_{\rho}$ ratio. This became manifest in a substantial ambiguity in the determination of $\bar{m}$ from the vector Ward identities. Indeed, there is a freedom of chiral extrapolation $(\chi \mathrm{E})$ on the lattice: at $\beta=5.6$. e.g., the extrapolation along the line of equal valence and sea quark hopping parameters, $\kappa^{\text {val }}=\kappa^{\text {sea }}$ (direct extrapolation), yields a value for $\bar{m}=m_{u / d}$ which is roughly a factor of two below the result from a semi-quenched, twostep extrapolation procedure. In the latter one first determines the values of $\kappa_{l}^{\text {val }}=\kappa_{l}^{v a l}\left(\kappa^{\text {sea }}\right)$ for each ensemble with fixed value of $\kappa^{\text {sea }}$ (similar to quenched simulations). For each $\kappa_{l}^{v a l}\left(\kappa^{\text {sea }}\right)$ $\bar{m}_{v a l}^{\overline{\mathrm{MS}}}\left(\kappa^{\text {sea }}\right)$ is computed and, subsequently, this function is extrapolated to the physical point $\kappa^{\text {sea }}=\kappa_{l}^{\text {sea }}$, at this given value of $\beta$.

Furthermore it has been shown by CP-PACS that the ambiguity seen at finite $a$ goes away after CE 2. CP-PACS has used an improved action that allowed them to go to rather coarse lattices, providing them with a long lever arm for the various CE. In the present context, their lever arm turned out to be of little value, however, since

\footnotetext{
*Talk presented by N. Eicker.
}

the extrapolation with a linear $\mathrm{CE}$ led them to a very poor $\chi^{2}$ with such an ansatz, invalidating the small error on their value of $\bar{m}$.

Given this situation we are motivated to resume the question with the standard Wilson fermionic action and attempt a scaling analysis, by complementing previous SESAM $/ \mathrm{T} \chi \mathrm{L}$ data with another set of vacuum configurations at $\beta=5.5$, as specified in table 1 .

\section{The light quark mass $\bar{m}$}

The determination of light quark masses can be sketched by the following steps:

(a) Fix $\kappa_{c}$ from the $\chi \mathrm{E}$ of $a m_{P S}^{2} \rightarrow 0$;

(b) Get $\kappa_{l}$ from $\frac{m_{P S}}{m_{V}} \rightarrow \frac{m_{\pi}}{m_{\rho}}$ using the $\chi \mathrm{E}$ of $a m_{V}$;

(c) Determine $a^{-1}$ via $r_{0} \simeq 0.5 \mathrm{fm}$;

(d) Compute the quark mass in the latticescheme:

$\bar{m}^{l a t}(a)=a^{-1} \frac{1}{2}\left(\frac{1}{\kappa_{l}}-\frac{1}{\kappa_{c}}\right) ;$

(e) Convert into $\overline{\mathrm{MS}}$-scheme using

$\bar{m}^{\overline{\mathrm{MS}}}(\mu=1 / a)=Z_{m} \bar{m}^{l a t}(a) ;$

(f) Rescale the quark mass to $\mu=2 \mathrm{GeV}$;

(g) Extrapolate $a \rightarrow 0$.

$Z_{M}$ within this work was determined using a tadpole improved perturbative procedure [5].

In the case of the above-quoted semi-quenched $\chi \mathrm{E}$ procedure the results for $\bar{m}^{\overline{\mathrm{MS}}}(2 \mathrm{GeV})$ have to be extrapolated to the physical mass of the sea quarks before sending $a \rightarrow 0$. 


\begin{tabular}{|c|c|c|c|c|c|c|c|c|}
\hline$\beta$ & $N_{s}^{3} \times N_{t}$ & $\kappa$ & $N_{t r a j}$ & $N_{\text {conf }}$ & $m_{P S} / m_{V}$ & $\left(a m_{P S} N_{s}\right)^{-1}$ & $r_{0} a^{-1}$ & $a[\mathrm{GeV}]$ \\
\hline \multirow[t]{4}{*}{5.5} & $16^{3} \times 32$ & 0.1580 & 4000 & 119 & $0.854(2)$ & $0.113(1)$ & $4.027(24)$ & $1.587(9)$ \\
\hline & & 0.1590 & 4500 & 140 & $0.803(9)$ & $0.142(1)$ & $4.386(26)$ & $1.728(10)$ \\
\hline & $a_{r_{0}}^{l}=0.092 \mathrm{fm}$ & 0.1596 & 5500 & 199 & $0.753(6)$ & $0.170(2)$ & $4.675(34)$ & $1.842(13)$ \\
\hline & & 0.1600 & 5500 & 200 & $0.675(10)$ & $0.204(3)$ & $4.889(30)$ & $1.926(12)$ \\
\hline \multirow[t]{6}{*}{$\overline{5.6}$} & $16^{3} \times 32$ & 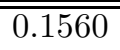 & 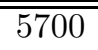 & 198 & "0.834(3) & $0.140(1)$ & 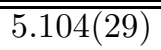 & $2.011(11)$ \\
\hline & & 0.1565 & 5900 & 208 & $0.812(9)$ & $0.156(2)$ & $5.283(52)$ & $2.08(2)$ \\
\hline & & 0.1570 & 6000 & 201 & $0.762(7)$ & $0.181(2)$ & $5.475(72)$ & $2.16(3)$ \\
\hline & & 0.1575 & 6500 & 206 & $0.684(8)$ & $0.223(3)$ & $5.959(77)$ & $2.35(3)$ \\
\hline & $24^{3} \times 40$ & 0.1575 & 5100 & 185 & $0.701(6)$ & $0.151(2)$ & $5.892(27)$ & $2.321(11)$ \\
\hline & $a_{r_{0}}^{l}=0.076 \mathrm{fm}$ & 0.1580 & 4500 & 158 & $0.566(14)$ & $0.209(4)$ & $6.230(60)$ & $2.45(2)$ \\
\hline
\end{tabular}

Table 1

Simulation parameters. $a$-values in 2nd column hold at $\kappa_{l}$. $\left(a m_{P S} N_{s}\right)^{-1}$ is discussed in [3] $r_{0}$ for $\beta=5.6$ from 肫, for $\beta=5.5$ using the same approach.

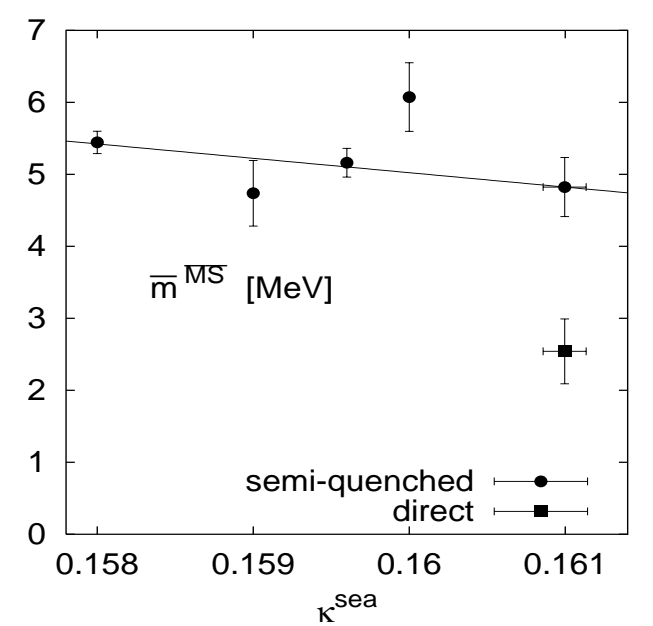

Figure 1. Light quark mass at $\beta=5.5$ in the $\overline{\mathrm{MS}}$-scheme at $\mu=2 \mathrm{GeV}$. Square-symbol from direct, circles from semi-quenched extrapolation.

In order to achieve appropriate statistical errors throughout this analysis one has to take into account the correlation between the observables. These correlations occur especially within the semi-quenched analysis, since all observables inside a $\chi \mathrm{E}$ are determined on the same set of configurations. Therefore all errors are determined using the jackknife procedure within all steps (a) to $(\mathrm{g})$ sketched above.

Fig. 2 shows the results for $\bar{m}$ in the $\overline{\mathrm{MS}}$-scheme for $\beta=5.6$ versus $\kappa^{\text {sea }}$. Obviously the semiquenched values for $\kappa=0.1575$ on the small lattice and for $\kappa=0.158$ on the larger lattice de-

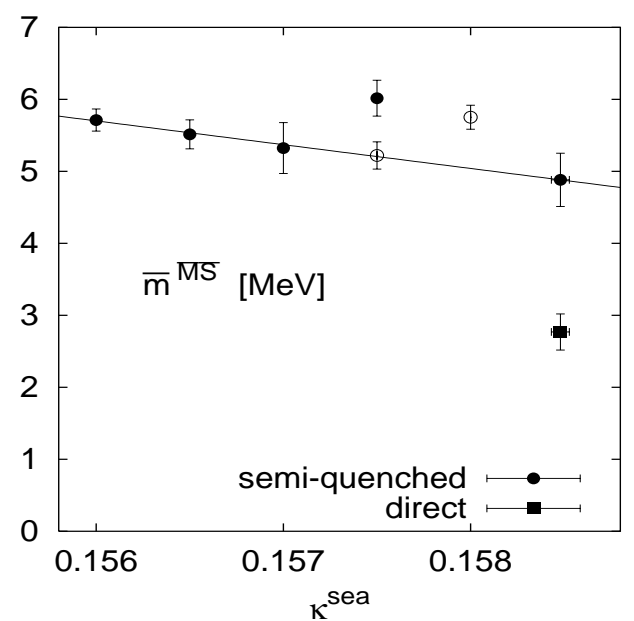

Figure 2. Same as in Fig. 1 1 but for $\beta=5.6$. Data with open symbols from larger lattices.

viate from the general behavior of the data. A more detailed investigation shows that this is due to finite-size effects. Hence these two points are neglected in the extrapolation to the physical sea quark mass. Furthermore we reconfirm the above mentioned discrepancy between the results for the semi-quenched and the direct extrapolations.

Fig. 1] displays the corresponding results for $\beta=5.5$. Here the value for $\kappa=0.160$ clearly shows finite-size effects and is hence discarded.

Fig. 3 presents the CE $a \rightarrow 0$, with $a$ as obtained from $r_{0}$ at $\kappa_{l}$. The continuum value for the light quark mass is determined using a constrained fit to the semi-quenched and direct val- 


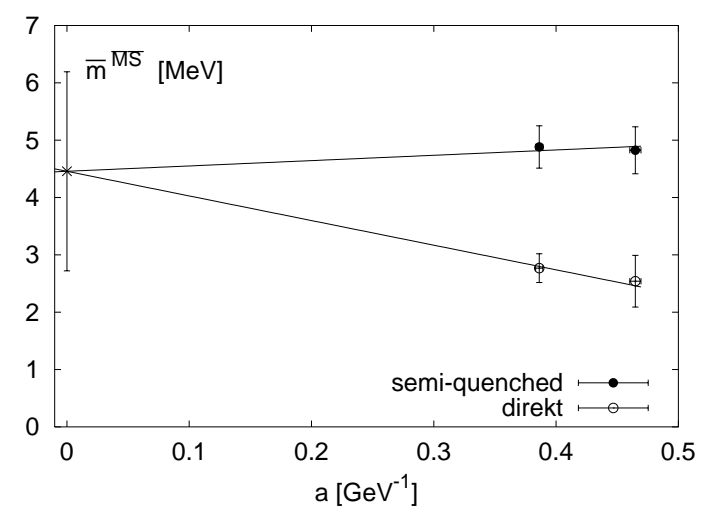

Figure 3. Extrapolating $\bar{m}$ in $a \rightarrow 0$.

ues at two lattice constants each. The fit is also shown in the figure.

As a final result for the light quark mass we get

$\bar{m}^{\overline{\mathrm{MS}}}(2 \mathrm{GeV})=4.5(1.7) \mathrm{MeV}$.

Within the bigger errors of our analysis, this number is in good agreement with the result quoted by CP-PACS [2].

\section{The strange quark mass $m_{s}$}

In contrast to the light quark mass there is no reasonable way to carry out a direct $\chi \mathrm{E}$ for the mass of the strange quark. This is due to the fact that directly extrapolated results would describe mesons with strange valence quarks within a sea of strange sea quarks. Indeed, nature is given by a sea of light sea quarks and therefore only the semi-quenched $\chi \mathrm{E}$ makes sense.

On the other hand there are several ways to determine $\kappa_{s}$, the hopping-parameter corresponding to the mass of the strange quark. In this analysis we investigated three different inputs: $\frac{m_{\phi}}{m_{\rho}}, \frac{m_{K}}{m_{\pi}}$ and $\frac{m_{K^{*}}}{m_{\rho}}$.

From a semi-quenched analysis of $m_{s}$, one obtains the results displayed in Fig. 4. The data clearly show deviations for the different definitions of the quark mass which are expected to disappear in the continuum. Hence again a constrained fit was done for the CE.

The result of this fit for the mass of the strange quark is

$m_{s}^{\overline{\mathrm{MS}}}(2 \mathrm{GeV})=92(83) \mathrm{MeV}$.

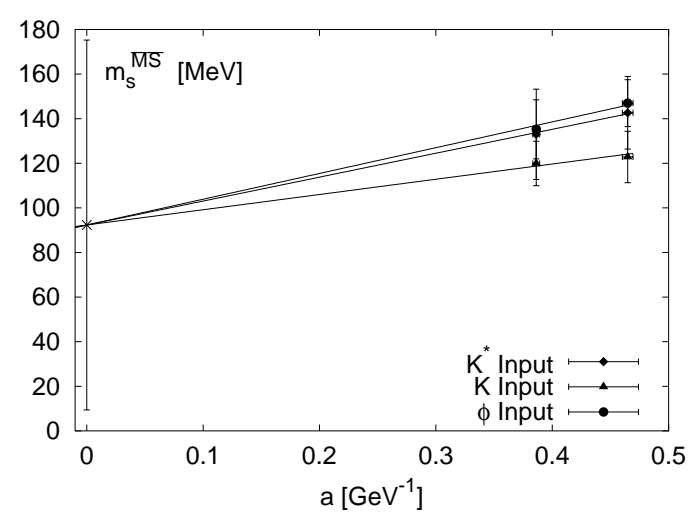

Figure 4. Extrapolating $m_{s}$ in $a \rightarrow 0$.

Obviously the strange quark mass needs all the more a larger range of $a$-values for a safe CE.

\section{Conclusions}

We conclude that $(i)$ statistical errors of 5 to $10 \%$ can be achieved before CE, (ii) with additional simulations at smaller $\beta$, a reliable $\bar{m}$ determination with $10 \%$ accuracy is within reach.

\section{Acknowledgments}

We thank G. Bali for computing the $r_{0}$ data, with support of the EC under contract HPRNCT-2000-00145 Hadrons/Lattice QCD. N.E. thanks DESY for partly supporting his PhD project. B.O. was supported by the DFG Graduiertenkolleg "Feldtheoretische Methoden in der Elementarteilchentheorie und Statistischen Physik".

\section{REFERENCES}

1. N. Eicker, et al., Phys. Lett. B407 (1997) 290.

2. A. Ali Khan et al., hep-lat/0105015 (2001).

3. B. Orth, et al., these proceedings.

4. B. Bolder et al., Phys. Rev. D62 (2000) 054503.

5. P. Lepage et al., Phys. Rev. D 48 (1993) 2250. 